\title{
Toll-like receptor 3 pathway restricts Marek's disease virus infection
}

\author{
Haitao Zou ${ }^{1,2}$, Ruixue Su ${ }^{1,2}$, Jing Ruan ${ }^{1,2}$, Hongxia Shao ${ }^{1,2,3}$, Kun Qian ${ }^{1,2,3}$, Jianqiang \\ Ye $^{1,2,3}$ and Aijian Qin ${ }^{1,2,3}$ \\ ${ }^{1}$ Ministry of Education Key Lab for Avian Preventive Medicine, Yangzhou University, Yangzhou, Jiangsu, 225009, P.R. China \\ ${ }^{2}$ Jiangsu Co-Innovation Center for Prevention and Control of Important Animal Infectious Diseases and Zoonoses, Yangzhou, \\ Jiangsu, 225009, P.R. China \\ 3 Jiangsu Key Lab of Zoonosis, Yangzhou, Jiangsu, 225009, P.R. China \\ Correspondence to: Aijian Qin, email: aijian@yzu.edu.cn \\ Keywords: Marek's disease virus, toll-like receptor 3, poly (l:C), interferon- $\beta$, inflammatory cytokines \\ Abbreviations: IRF3: interferon regulatory factor3, IKKa: IKB kinase a, TANK: TRAF family member-associated NFKB activator \\ Received: April 04, $2017 \quad$ Accepted: July 12, $2017 \quad$ Published: August 07, 2017 \\ Copyright: Zou et al. This is an open-access article distributed under the terms of the Creative Commons Attribution License 3.0 \\ (CC BY 3.0), which permits unrestricted use, distribution, and reproduction in any medium, provided the original author and source \\ are credited.
}

\section{ABSTRACT}

Marek's disease virus (MDV) is an $\alpha$-herpesvirus that causes immune suppression and T lymphoma in chickens. Toll-like receptor 3 (TLR3) is critical for the host immune response against MDV infection. Previously, our team demonstrated that pre-treatment of TLR3 agonist poly (I:C) inhibited Marek's disease virus infection in chicken embryo fibroblasts (CEFs). However, whether TLR3 inhibits the aggravation of MDV infection is unknown. In the current study, we found that TLR3 activation in MDV-infected CEFs effectively inhibited virus spread. Using pharmacological approaches, we revealed that pro-inflammatory cytokines and interferon- $\beta$ induced by TLR 3 could restrict Marek's disease virus infection. This study contributes to elucidating the function and mechanism of the TLR3 pathway in host immune responses against MDV infection.

\section{INTRODUCTION}

The innate immune system recognizes pathogenassociated molecular patterns (PAMPs) through pattern recognition receptors (PRRs). Toll-like receptors (TLRs) are a typical class of PRRs in the innate immune response against viral infections. Toll-like receptor 3 (TLR3), a member of the TLR family, is a type I intracellular transmembrane protein that contains a large leucinerich repeat (LRR) in its extracellular region and a TollIL-1 receptor (TIR) homology signalling domain in its cytoplasmic region [1]. Double-stranded RNA (dsRNA) binding leads to TLR3 dimerization and activation of its TIR domain, which recruits the adapter molecule TIRcontaining adaptor molecule-1 (TICAM-1; also called TRIF) [2]. TRIF recruits either TNF receptor-associated factor 6 (TRAF6) and receptor-interacting protein 1 (RIP1) to activate NF- $\mathrm{kB}$, or TRAF3 to induce interferon regulatory factor 3 (IRF3), mediating innate immune responses to limit viral replication [3].

An increasing body of evidence has indicated that TLR3 responds to dsRNA derived from viral particles or infected cells and subsequently initiates signal transduction to induce a cytokine response, such as the production of interleukelin-6 (IL-6) and interferon- $\beta$ (IFN- $\beta$ ) $[4,5]$. Activation of TLR3 impairs the replication of several RNA viruses, including dengue virus (DENV), human immunodeficiency virus (HIV), influenza virus and respiratory syncytial virus (RSV), in different cell types through induction of cytokines and interferon [6-9]. In studies of herpesvirus, stimulation with TLR3 agonist was also found to inhibit herpes simplex virus (HSV) replication in human neuronal cells and genital epithelial cells $[10,11]$. Furthermore, pretreatment with a TLR3 agonist induced innate immunity against HSV infection in a mouse model $[12,13]$. Marek's disease virus (MDV) is an avian $\alpha$-herpesvirus that causes immune suppression and T lymphoma in chickens [14]. MDV is a strict cellassociated virus and, thus, has a long and complex viral replication cycle [15]. MDV infection was reported to be inhibited in chicken embryo fibroblasts (CEFs) pretreated with poly (I:C), a TLR3 agonist [16]. However, it is unknown whether activation of TLR3 can restrict the aggravation of MDV infection. 
In the present study, we first showed that activation of TLR3 inhibited the aggravation of MDV infection. We then demonstrated that both IFN- $\beta$ and pro-inflammatory cytokines induced by TLR3 activation contributed to the restriction of MDV infection in CEFs.

\section{RESULTS}

\section{Activation of TLR3 restricted the development of MDV infection}

In MDV-infected CEFs, we found that TLR3 activation effectively restricted the development of MDV infection. The TLR3 agonist poly (I:C) was added to infected cells at 24,48 and $72 \mathrm{hpi}$, respectively. At a

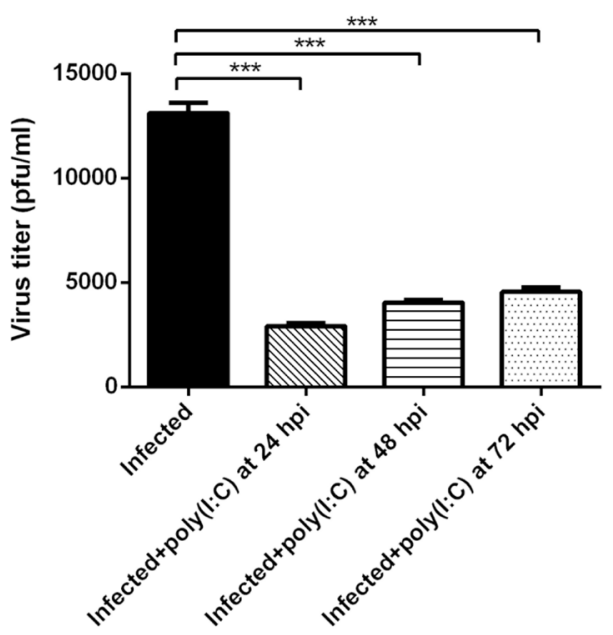

C

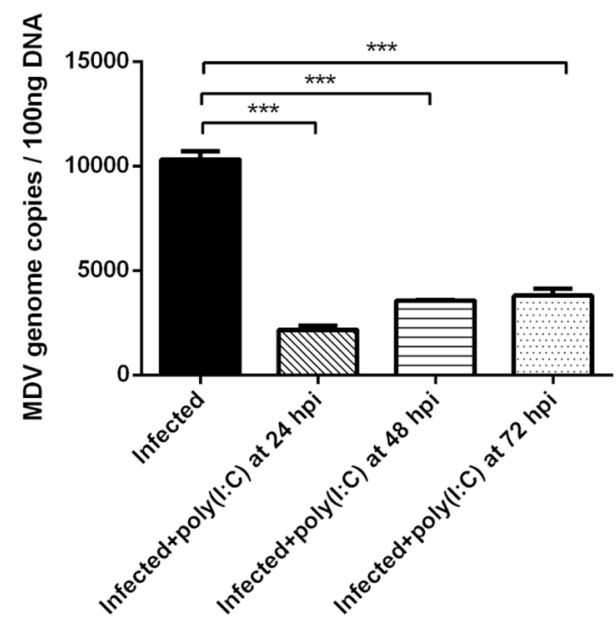

$96 \mathrm{hpi}$, viral titre and viral genomic copies in infected CEFs stimulated with poly (I:C) for 24,48 and $72 \mathrm{~h}$ were significantly lower than those in the un-stimulated group (Figure 1a, 1c). At the same time, the plaques size in infected CEFs stimulated with poly (I:C) for 24, 48 and $72 \mathrm{~h}$ were also smaller than those in the control group (Figure 1b). Furthermore, western blot assays showed that the expression of the viral protein $\mathrm{gB}$ in infected CEFs stimulated with poly (I:C) for 24,48 and $72 \mathrm{~h}$ was less than that in the untreated group (Figure 1d). Similar inhibitory effect of poly (I:C) were found in CEFs infected with MDV virulent strain GA (data not shown). These results suggested that TLR3 activation restricted MDV infection regardless of when that activation occurred.

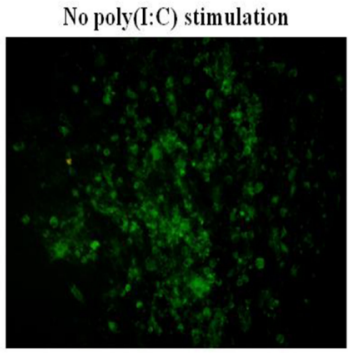

Poly(I:C) stimulation at $24 \mathrm{hpi}$

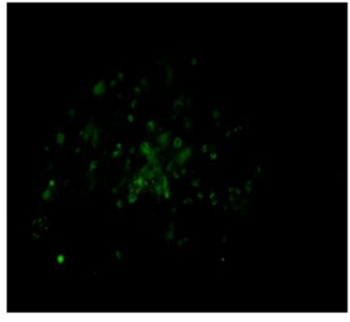

Poly(I:C) stimulation at $48 \mathrm{hpi}$
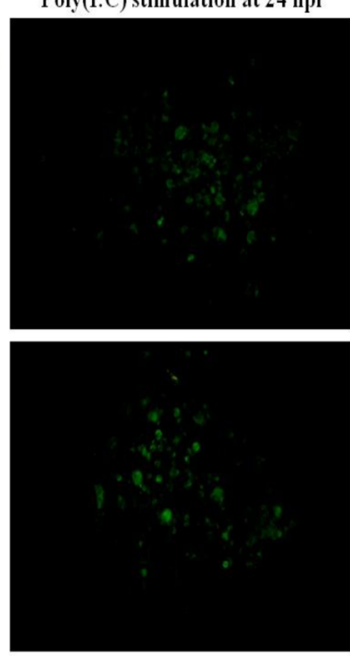

Poly(I:C) stimulation at $72 \mathrm{hpi}$ d

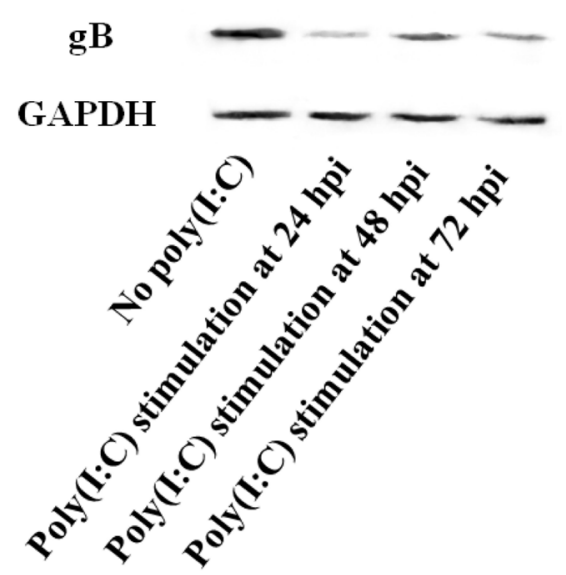

Figure 1: The effect of TLR3 activation on MDV infection. (a) Viral titre of the RB1B strain in CEF cells either untreated or stimulated with poly (I:C) at 96 hpi. (b) Plaques of the RB1B strain in CEF cells either untreated or stimulated with poly (I:C) at 96 hpi. (c) Viral genome copies of the RB1B strain in CEF cells either untreated or stimulated with poly (I:C) at 96 hpi. (d) Level of the viral protein $\mathrm{gB}$ in the RB1B strain in CEF cells either untreated or stimulated with poly (I:C) at 96 hpi. An asterisk $(*)$, double asterisk $(* *)$ or triple asterisk $(* * *)$ indicates $\mathrm{p}<0.05,0.01<\mathrm{p}<0.05$ or $\mathrm{p}<0.01$ for statistical difference from the controls, respectively. Error bars represent standard errors. 


\section{Poly (I:C) stimulation increased transcripts} associated with the TLR3 pathway in MDVinfected CEFs

We investigated the expression pattern of several genes associated with the TLR3 pathway by realtime PCR. TLR3, IRF3 and IKKa were substantially increased in the stimulated groups compared with those in the control group (Figure 2a-2c). TLR3 increase in the stimulated groups was further confirmed by western blot analysis (Figure $2 \mathrm{~g}$ ). IRF3 and IKK $\alpha$ were related to IFN- $\beta$ and inflammatory cytokine production, respectively. Similarly, the mRNA levels of $I F N-\beta, I L-6$ and $T N F$ - $\alpha$ were significantly increased in MDV-infected CEFs stimulated with poly (I:C) (Figure 2d-2f). These data suggested that TLR3 activation induced IFN- $\beta$ and inflammatory cytokines to restrict MDV infection.

\section{Both IFN- $\beta$ and inflammatory cytokines induced by TLR3 contributed to MDV infection inhibition}

To confirm that the anti-MDV effect was mediated by IFN- $\beta$ and inflammatory cytokines, we used two specific small-molecule inhibitors, BX795 and resveratrol, to interfere with the induction of a

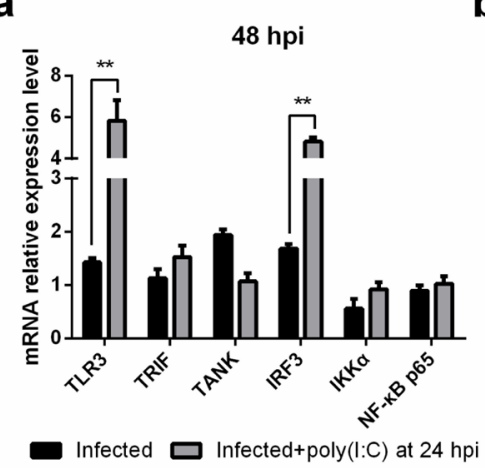

b

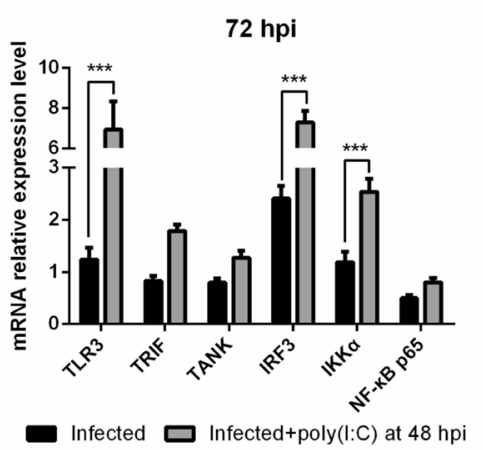

C

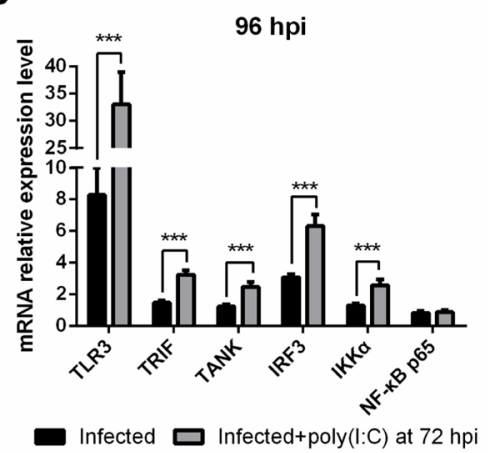

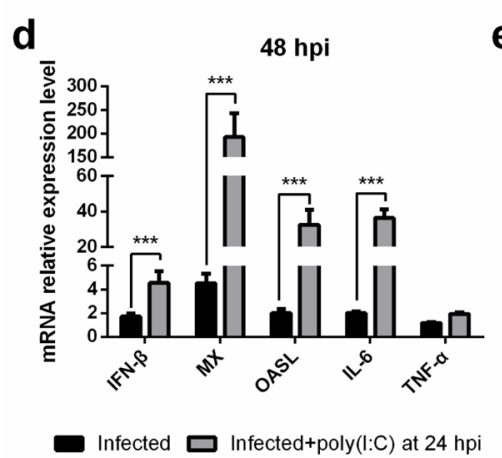
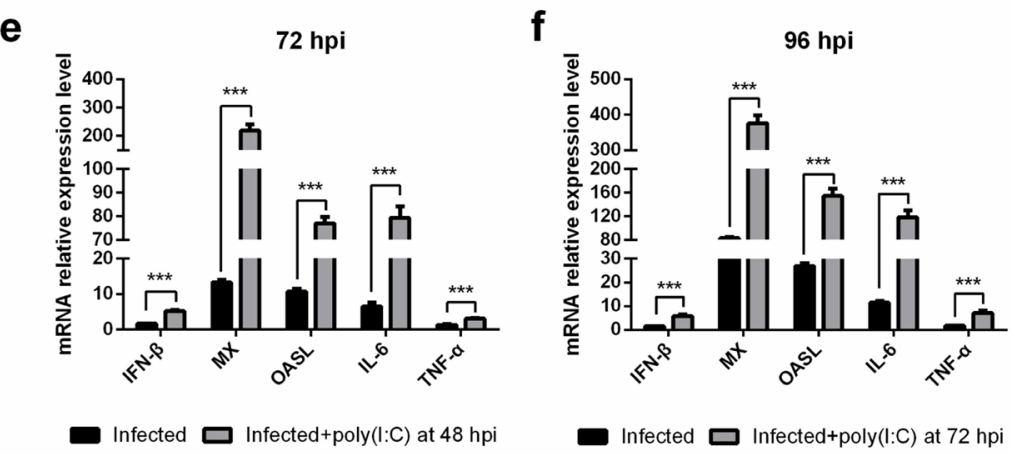

g

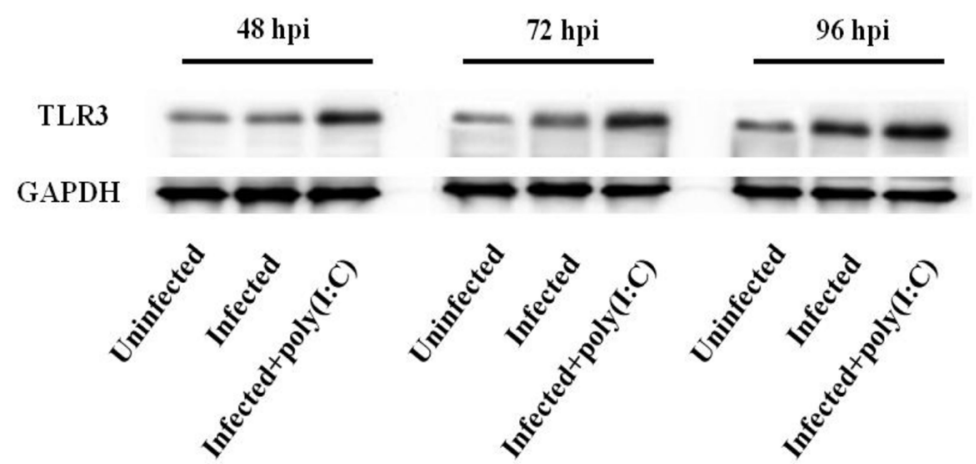

Figure 2: The effect of TLR3 activation on expression of genes associated with the TLR3 pathway in MDV-infected CEF Cells. Transcriptional level of genes associated with the TLR3 pathway in MDV-infected CEFs either untreated or stimulated with poly (I:C) at 48 hpi (a, d), 72 hpi (b, e) and 96 hpi (c, f). (g) Level of TLR3 protein in MDV-infected CEFs either untreated or stimulated with poly (I:C) at $48 \mathrm{hpi}, 72 \mathrm{hpi}$ and 96 hpi. An asterisk (*), double asterisk $(* *)$ or triple asterisk (***) indicates $\mathrm{p}<0.05,0.01<\mathrm{p}<0.05$ or $\mathrm{p}<0.01$ for statistical difference from the controls, respectively. Error bars represent standard errors. 
IFN- $\beta$ and inflammatory cytokines, respectively. The BX795 at a concentration of $10 \mu \mathrm{M}$ of and resveratrol at a concentration of $100 \mu \mathrm{M}$ showed no significant negative effect on CEFs in CCK8 assay (Figure 3a). And the results of ELISA and western blot confirmed that BX795 and resveratrol were able to effectively inhibit the production of IFN- $\beta$ and inflammatory cytokines, respectively (Figure 3a). Viral titre and number of viral genome copies showed that both BX795 and resveratrol attenuated rather than abolished the antiviral effect of poly (I:C) (Figure 3b, 3c). At the same time, the plaques in the inhibiter-treated groups were larger than those in the untreated group, but smaller than those in the group infected with MDV only (Figure 3d). Similar results were also found in the viral gene expression analysis (Figure 3e). Overall, the data suggested that IFN- $\beta$ and inflammatory cytokines induced by the TLR3 pathway contributed to inhibiting MDV infection.

\section{DISCUSSION}

TLR3 has been identified as an important innate immune-recognition receptor for dsRNA. Many studies have shown the potential antiviral function of TLR3 in herpesvirus infection [10-12, 17]. Previously, we demonstrated that MDV infection was inhibited in CEFs pretreated with poly (I:C) [16]. Moreover, our current study showed that activation of TLR3 also restricted the development of MDV infection. Thus, TLR3 activation is a key mediator of the host immune response against MDV regardless of when that activation occurred.

Binding of TLR3 and dsRNA promotes signal transduction through the adapter protein TRIF to activate the transcription factors IRF3 and NF- $\kappa \mathrm{B}$, which promote the expression of IFN- $\beta$ and inflammatory cytokines. Several studies have shown that inflammatory cytokine and IFN- $\beta$ induction by TLRs can help the host impair DENV- a
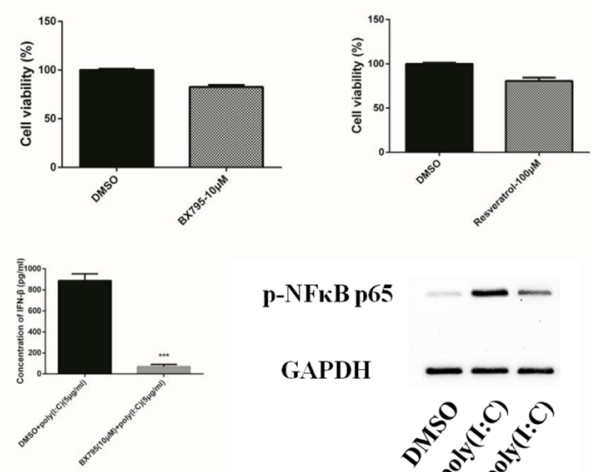

d
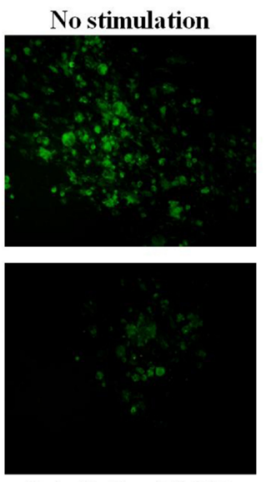

Poly(I:C) + DMSO
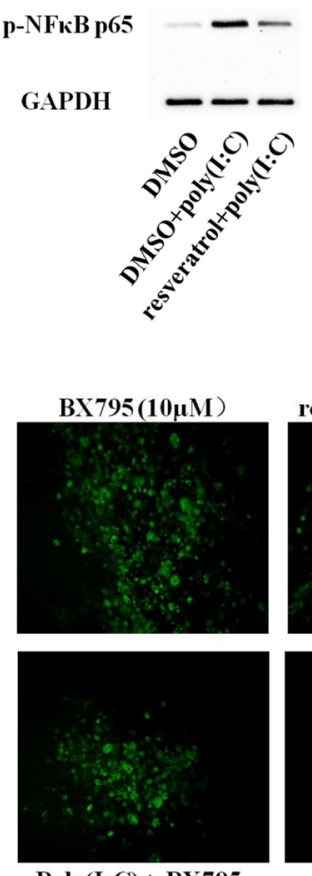

Poly(I: C) + BX795 b

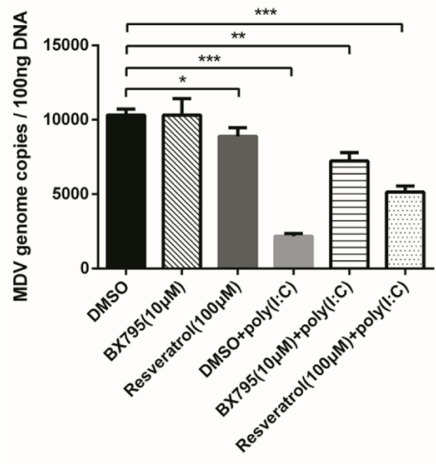

C

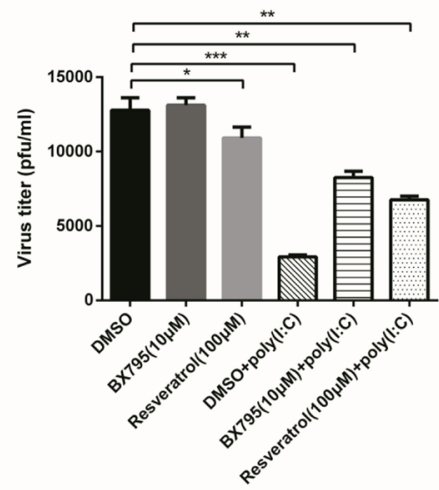

e

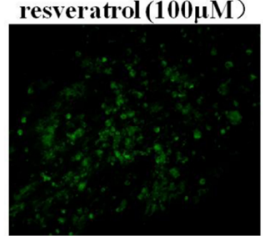

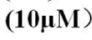

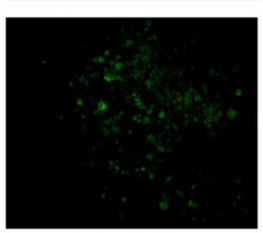

$\operatorname{Poly}(\mathbf{I}: \mathbf{C})+$ resveratrol $(100 \mu \mathrm{M})$

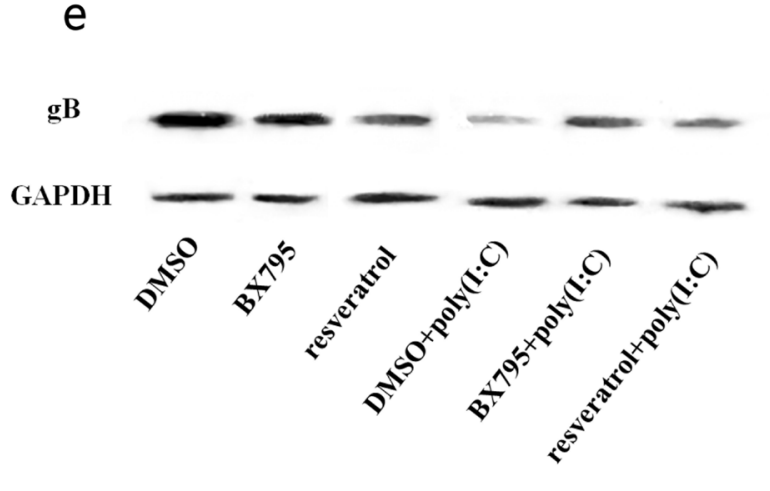

Figure 3: TLR3 activation induced IFN- $\beta$ and inflammatory cytokines to restrict MDV infection. (a) The inhibitory effect and cell viability of two inhibitors in CEFs. (b) Viral titre of the RB1B strain in poly (I:C)-stimulated CEF cells either untreated or treated with inhibitors at 96 hpi. (c) Viral genome copies of the RB1B strain in poly (I:C)-stimulated CEF cells either untreated or treated with inhibitors at 96 hpi. (d) Plaques of the RB1B strain in poly (I:C)-stimulated CEF cells either untreated or treated with inhibitors at 96 hpi. (e) Level of the viral protein $\mathrm{gB}$ in the RB1B strain in poly (I:C)-stimulated CEF cells either untreated or treated with inhibitors at 96 hpi. An asterisk (*), double asterisk $(* *)$ or triple asterisk $(* * *)$ indicates $\mathrm{p}<0.05,0.01<\mathrm{p}<0.05$ or $\mathrm{p}<0.01$ for statistical difference from the controls, respectively. Error bars represent standard errors. 
2, HIV, and RSV infections [6-8]. BX795 is a relatively specific inhibitor of TBK1 and IKKE which block the phosphorylation, nuclear translocation, and transcriptional activity of IRF3, to inhibit the production of IFN- $\beta$ [18]. Resveratrol specifically inhibits TRIF signaling in the TLR3 pathway by targeting TBK1 and RIP1 in TRIF complex, resulting in inhibition of pro-inflammatory cytokines expression [19]. In chemical inhibitors assays, our results indicated that suppressing either inflammatory cytokines or IFN- $\beta$ significantly attenuated the antiMDV effect of poly (I:C). These data suggested that the antiviral effects of TLR3 in MDV infection are through the production of IFN- $\beta$ and inflammatory cytokines. Previous studies showed that type I IFN protected cell and host from MDV infection, which was consistent with our finding [20, 21]. Several IFN-stimulated genes (ISG) induced by type I IFN have been identified and characterized in chicken [22]. The IFN positive-feedback loop is important for inducing and sustaining antiviral innate immune responses. In this study, we observed that synthetic dsRNA stimulation elevated two typical ISGs, Mx and OASL, in MDVinfected CEFs. These results indicated that TLR3 was involved in the IFN- $\beta$ response.

Moreover, no significant change in $I F N-\beta$ expression was detected during MDV infection, while IFN- $\beta$ was increased in MDV-infected CEFs after poly (I:C) stimulation. These data suggested that MDV inhibits IFN- $\beta$ production. Several studies have identified a variety of mechanisms for blocking IFN- $\beta$ production during $\alpha$-herpesvirus infection. The infected cell protein 0 encoded by HSV-1 inhibits IRF3- and IRF7-mediated activation of the IFN- $\beta$ gene [23]. The HSV-1 ubiquitin-specific protease UL36 and serine/threonine kinase US3 inhibit IFN- $\beta$ production by deubiquitinating TRAF3 and hyperphosphorylating IRF3, respectively $[24,25]$. The MDV genes located in the UL and US segments are largely homologous to, and arranged collinearly with, those of HSV-1 [15]. Thus, there are likely to be similar mechanisms for blocking IFN- $\beta$ production during MDV infection, which can further explain the immune suppression caused by this infection.

Inflammatory cytokines are indispensable in host defence against $\alpha$-herpesvirus. IL-18 is associated with the rapid activation of NK cells to restrict HSV1 replication in the lung [26]. Mast cells inhibit HSV-1 infection through TNF- $\alpha$ and IL-6 production [27], and IL-6 deficiency enhances susceptibility to HSV-1 in mice [28]. Similarly, IL-1 $\beta$, IL-6 and IL-18 were associated with an immune response to MDV, but this has yet not been directly confirmed. The current results indicated that inflammatory cytokines induced by TLR3 inhibit MDV infection. Moreover, we revealed that inflammatory cytokines were directly related to the anti-MDV effects. Unfortunately, it is unclear which type of inflammatory cytokine most efficiently restricts MDV infection. Several chicken inflammatory cytokines have been expressed with biological activity in vitro $[29,30]$. Thus, it is possible to test the effect of different inflammatory cytokines on MDV infection, which may promote the development of novel MDV vaccines or anti-MDV drugs.

Collectively, our results have helped elucidate the function and mechanism of the TLR3 pathway in the host immune response against MDV.

\section{MATERIALS AND METHODS}

\section{Cells and virus}

Primary CEFs were prepared from 10-day-old specific-pathogen-free (SPF) embryos obtained from Merial Vital (Laboratory Animal Technology Co., Ltd, Beijing, China) and cultured in DMEM supplemented with $5 \% \mathrm{FBS}$ at $37^{\circ} \mathrm{C}$ in $5 \% \mathrm{CO}_{2}$. The very virulent $\mathrm{RB} 1 \mathrm{~B}$ strain of MDV was maintained in our laboratory.

\section{Reagents and antibodies}

The TLR3 agonist poly (I:C) (HMW) and the inhibitors (BX795 and resveratrol) were purchased from InvivoGen (San Diego, CA, USA). Mouse monoclonal anti-GAPDH antibody was from Abcam (Cambridge, MA, USA). Rabbit polyclonal anti-TLR3 antibody was from Novus Biologicals (Littleton, Colorado, USA). The mouse anti-MDV monoclonal antibody BD8 and rabbit polyclonal anti-NFKB antibody were maintained by our laboratory. The ELISA kit for chicken IFN- $\beta$ was from Nanjing Senbeijia Biological Technology Co., Ltd (Nanjing, China).

\section{Virus infection and cell treatment}

Secondary CEFs were seeded in 6-well plates and cultured for 18-24 $\mathrm{h}$ following infection with the RB1B strain at an $\mathrm{MOI}=0.001$. The TLR3 agonist poly $(\mathrm{I}: \mathrm{C})$ was added at a final concentration of $5 \mu \mathrm{g} / \mathrm{ml}$ to infected cells at 24, 48 and $72 \mathrm{~h}$ post infection (hpi). The infected cells were collected at 48, 72 and 96 hpi [16].

Secondary CEFs infected with the RB1B strain at an $\mathrm{MOI}=0.01$ were cultured for $24 \mathrm{~h}$. MDV-infected CEFs were pretreated with inhibitors (either $10 \mu \mathrm{M}$ BX795 or $100 \mu \mathrm{M}$ resveratrol) for $2 \mathrm{~h}$ following stimulation with poly (I:C). The infected cells were collected at $96 \mathrm{hpi}$.

\section{Real-time PCR}

The total RNA and MDV genomic DNA from infected cells were isolated using TRIzol Reagent (Invitrogen) following the manufacturer's instructions. The cDNA synthesis was performed using a PrimeScript ${ }^{\mathrm{TM}}$ RT reagent Kit (TaKaRa, Dalian, China). Real-time PCR was performed using SYBR Premix ExTaq II (TaKaRa Dalian, China) in an ABI 7500 real-time PCR system (Applied Biosystems, CA, USA). The sequences of specific primers used for real-time PCR were previously 
published elsewhere [31]. Gene expression levels were normalised to the expression of chicken 18S snRNA. The changes in gene expression were calculated using the $2^{-\Delta \Delta \mathrm{Ct}}$ method.

\section{Virus titration}

The infected cells were centrifuged and resuspended in $1 \mathrm{ml}$ medium. Infected-cell suspension was made for serial 10 -fold dilution. Secondary CEFs were seeded in 96-well plates and cultured over night, and then these cells were infected with MDV in different dilution. Each dilution group has twelve replicates. After incubation at $37^{\circ} \mathrm{C}$ in $5 \% \mathrm{CO}_{2}$ for $96 \mathrm{~h}$, the cells were treated as described in plaque assay. The numbers of plaques in different dilution were counted.

\section{Virus Plaque compared by Immunofluorescence}

The cells were fixed with an acetone: ethanol solution (3:2) for $10 \mathrm{~min}$ and washed once with PBS. After the fixed cells were blocked with $1 \%$ BSA in PBS, they were incubated with the mouse anti-MDV monoclonal antibody BD8 for $45 \mathrm{~min}$ at $37^{\circ} \mathrm{C}$. After three washes with PBS, the cells were incubated with FITC-conjugated goat anti-mouse IgG (Sigma-Aldrich, USA) for another $45 \mathrm{~min}$. After three washes with PBS, the cells were examined under a fluorescence microscope. The plaque sizes between different groups were compared.

\section{Western blot analysis}

Cell pellets were washed once with PBS and lysed with RIPA buffer containing protease inhibitors on ice. The samples were loaded with $5 \times$ denaturing sample buffer and separated by $12 \%$ SDS-PAGE. The proteins were transferred to a $0.2-\mu \mathrm{m}$ nitrocellulose $(\mathrm{NC})$ membrane. The mouse monoclonal anti-GAPDH antibody, rabbit polyclonal anti-TLR3 antibody, rabbit polyclonal anti-NFKB antibody and mouse anti-MDV monoclonal antibody BD8 were used as primary antibodies, while either HRP-conjugated goat anti-mouse IgG or HRPconjugated goat anti-rabbit IgG (Sigma-Aldrich, USA) served as the secondary antibody. Finally, the membranes were washed and visualized using chemiluminescence (Protein Simple, Fluorchem E FE0605).

\section{Statistical analysis}

The data were analysed using the Student's $t$ test and GraphPad PRISM 6 software. P values $<0.05$ was considered significant.

\section{Author contributions}

HTZ and AJQ conceived and designed the experiments; HTZ and RXS performed the experiments;
HTZ, AJQ, KQ, HXS and JQY analyzed the data; HTZ, JR, KQ and HXS contributed reagents/materials/analysis tools. HTZ and AJQ contributed to the writing of the manuscript. HTZ prepared figures. All authors reviewed the manuscript.

\section{CONFLICTS OF INTEREST}

The authors declare no competing financial interests.

\section{FUNDING}

This research was supported by the National Natural Science Foundation of China (31472192), The National Key Research and Development Program of China (2016YFD0500803), the Special Foundation for State Basic Research Program of China (2013FY113300-4) and the Priority Academic Program Development of Jiangsu Higher Education Institutions.

\section{REFERENCES}

1. Jin MS, Lee JO. Structures of the toll-like receptor family and its ligand complexes. Immunity. 2008; 29:182-191.

2. Jiang Z, Mak TW, Sen G, Li X. Toll-like receptor 3-mediated activation of NF-kappaB and IRF3 diverges at Toll-IL-1 receptor domain-containing adapter inducing IFN-beta. Proc Natl Acad Sci U S A. 2004; 101:3533-3538.

3. Kawai T, Akira S. SnapShot: Pattern-recognition receptors. Cell. 2007; 129:1024.

4. Takeuchi O, Akira S. Innate immunity to virus infection. Immunological reviews. 2009; 227:75-86.

5. Perales-Linares R, Navas-Martin S. Toll-like receptor 3 in viral pathogenesis: friend or foe? Immunology. 2013; 140:153-167.

6. Liang Z, Wu S, Li Y, He L, Wu M, Jiang L, Feng L, Zhang P, Huang X. Activation of Toll-like receptor 3 impairs the dengue virus serotype 2 replication through induction of IFN-beta in cultured hepatoma cells. PLoS One. 2011; 6:e23346.

7. Trapp S, Derby NR, Singer R, Shaw A, Williams VG, Turville SG, Bess JW Jr, Lifson JD, Robbiani M. Double-stranded RNA analog poly (I:C) inhibits human immunodeficiency virus amplification in dendritic cells via type I interferon-mediated activation of APOBEC3G. J Virol. 2009; 83:884-895.

8. Boukhvalova MS, Sotomayor TB, Point RC, Pletneva LM, Prince GA, Blanco JC. Activation of interferon response through toll-like receptor 3 impacts viral pathogenesis and pulmonary toll-like receptor expression during respiratory syncytial virus and influenza infections in the cotton rat Sigmodon hispidus model. J Interferon Cytokine Res. 2010; $30: 229-242$. 
9. Lau YF, Tang LH, Ooi EE, Subbarao K. Activation of the innate immune system provides broad-spectrum protection against influenza A viruses with pandemic potential in mice. Virology. 2010; 406:80-87.

10. Nazli A, Yao XD, Smieja M, Rosenthal KL, Ashkar AA, Kaushic C. Differential induction of innate anti-viral responses by TLR ligands against Herpes simplex virus, type 2 , infection in primary genital epithelium of women. Antiviral Res. 2009; 81:103-112.

11. Zhou Y, Ye L, Wan Q, Zhou L, Wang X, Li J, Hu S, Zhou $\mathrm{D}$, Ho W. Activation of Toll-like receptors inhibits herpes simplex virus-1 infection of human neuronal cells. J Neurosci Res. 2009; 87:2916-2925.

12. Herbst-Kralovetz MM, Pyles RB. Quantification of poly (I:C)-mediated protection against genital herpes simplex virus type 2 infection. J Virol. 2006; 80:9988-9997.

13. Boivin N, Sergerie Y, Rivest S, Boivin G. Effect of pretreatment with toll-like receptor agonists in a mouse model of herpes simplex virus type 1 encephalitis. J Infect Dis. 2008; 198:664-672.

14. Haq K, Schat KA, Sharif S. Immunity to Marek's disease: where are we now? Dev Comp Immunol. 2013; 41:439-446.

15. Osterrieder N, Kamil JP, Schumacher D, Tischer BK, Trapp S. Marek's disease virus: from miasma to model. Nature reviews Microbiology. 2006; 4:283-294.

16. Hu X, Zou H, Qin A, Qian K, Shao H, Ye J. Activation of Toll-like receptor 3 inhibits Marek's disease virus infection in chicken embryo fibroblast cells. Arch Virol. 2016; 161:521-528.

17. Prehaud C, Megret F, Lafage M, Lafon M. Virus infection switches TLR-3-positive human neurons to become strong producers of beta interferon. J Virol. 2005; 79:12893-12904.

18. Clark K, Plater L, Peggie M, Cohen P. Use of the pharmacological inhibitor BX795 to study the regulation and physiological roles of TBK1 and IkappaB kinase epsilon: a distinct upstream kinase mediates Ser-172 phosphorylation and activation. J Biol Chem. 2009; 284:14136-14146.

19. Youn HS, Lee JY, Fitzgerald KA, Young HA, Akira S, Hwang DH. Specific inhibition of MyD88-independent signaling pathways of TLR3 and TLR4 by resveratrol: molecular targets are TBK1 and RIP1 in TRIF complex. J Immunol. 2005; 175:3339-3346.

20. Volpini LM, Calnek BW, Sneath B, Sekellick MJ, Marcus PI. Interferon modulation of Marek's disease virus genome expression in chicken cell lines. Avian Dis. 1996; 40:78-87.
21. Volpini LM, Calnek BW, Sekellick MJ, Marcus PI. Stages of Marek's disease virus latency defined by variable sensitivity to interferon modulation of viral antigen expression. Vet Microbiol. 1995; 47:99-109.

22. Goossens KE, Ward AC, Lowenthal JW, Bean AG. Chicken interferons, their receptors and interferon-stimulated genes. Dev Comp Immunol. 2013; 41:370-376.

23. Lin R, Noyce RS, Collins SE, Everett RD, Mossman KL. The herpes simplex virus ICP0 RING finger domain inhibits IRF3- and IRF7-mediated activation of interferonstimulated genes. J Virol. 2004; 78:1675-1684.

24. Wang S, Wang K, Li J, Zheng C. Herpes simplex virus 1 ubiquitin-specific protease UL36 inhibits beta interferon production by deubiquitinating TRAF3. J Virol. 2013; 87:11851-11860.

25. Wang S, Wang K, Lin R, Zheng C. Herpes simplex virus 1 serine/threonine kinase US3 hyperphosphorylates IRF3 and inhibits beta interferon production. J Virol. 2013; 87:12814-12827.

26. Reading PC, Whitney PG, Barr DP, Wojtasiak M, Mintern JD, Waithman J, Brooks AG. IL-18, but not IL-12, regulates NK cell activity following intranasal herpes simplex virus type 1 infection. J Immunol. 2007; 179:3214-3221.

27. Aoki R, Kawamura T, Goshima F, Ogawa Y, Nakae S, Nakao A, Moriishi K, Nishiyama Y, Shimada S. Mast cells play a key role in host defense against herpes simplex virus infection through TNF-alpha and IL-6 production. J Invest Dermatol. 2013; 133:2170-2179.

28. LeBlanc RA, Pesnicak L, Cabral ES, Godleski M, Straus SE. Lack of interleukin-6 (IL-6) enhances susceptibility to infection but does not alter latency or reactivation of herpes simplex virus type 1 in IL-6 knockout mice. J Virol. 1999; 73:8145-8151.

29. Weining KC, Sick C, Kaspers B, Staeheli P. A chicken homolog of mammalian interleukin-1 beta: cDNA cloning and purification of active recombinant protein. European journal of biochemistry / FEBS. 1998; 258:994-1000.

30. Schneider K, Klaas R, Kaspers B, Staeheli P. Chicken interleukin-6. cDNA structure and biological properties. European journal of biochemistry / FEBS. 2001; 268:4200-4206.

31. Hu X, Xu W, Qin A, Wu G, Qian K, Shao H, Ye J. Marek's disease virus may interfere with T cell immunity by TLR3 signals. Veterinary research communications. 2014; 38:149-156. 